\title{
Ultrastructural Investigations of Arterial Bypass Conduits after the Use of Different Harvesting Techniques Using an Electron Microscope
}

\author{
İhsan Sami Uyar, MD, ${ }^{1}$ Dogan Kahraman, MD $^{2}$ Gökhan Keskin, MD,${ }^{3}$ Emced Khalil, MD ${ }^{4}$ \\ ${ }^{1}$ Department of Cardiothoracic Surgery, Ahi Evren University, Kirsehir, Turkey; ${ }^{2}$ Department of Cardiothoracic Surgery, Gaziantep \\ University School of Medicine, Gaziantep, Turkey; ${ }^{3}$ Division of Cardiology, Department of Medicine, Amasya University School of \\ Medicine, Amasya, Turkey; ${ }^{4}$ Department of Cardiothoracic Surgery, Gaziantep University School of Medicine, Gaziantep, Turkey
}

\section{ABSTRACT}

Objectives: The aim of this study was to perform morphometric analysis of arterial conduits harvested by harmonic scalpel in coronary artery bypass grafting $(\mathrm{CABG})$ patients.

Methods: From 100 CABG patients, 200 arterial conduits -100 radial arteries (RAs) and 100 left internal thoracic artery (LITAs) — were harvested. The patients had similar characteristics (mean age, sex ratio, comorbidities, etc.). We divided the patients into 2 groups according to harvesting technique. In group 1, a harmonic scalpel was used in 50 patients for harvesting arterial conduits (50 LITA and 50 RA). In group 2, conduits were harvested using low-voltage electrocautery. To prevent side effects of clipping, all conduits in both groups remained in perfused condition until anastomosis. A 10-mm length of conduit was cut for transmission electron microscopy investigation. We calculated duration of harvesting, blood flow changes, and histopathologic changes of the conduits according to a vessel scoring system.

Results: In the harmonic scalpel group, we detected pathologic findings - corruption of endothelial integrity, subendothelial damage, and endothelial pathology-in 5 specimens (10\%) (3 LITA [6\%] and 2 RA [4\%]). In group 2, pathologic findings were detected in 16 conduits $(32 \% ; 11$ LITA, $22 \%$, and 5 RA, 10\%). Endothelial dissection, subendothelial disarrangement, cellular separation due to intercellular edema, and subadventitial hematoma were the main pathologic changes in the classic harvesting method. There was a significant difference between the groups $(P=.001)$. Harvesting time of LITA was nearly similar in both groups: $26.9 \pm 11.1 \mathrm{~min}$ (range 25-38) in group 1 and $21.3 \pm 8.6 \mathrm{~min}$ (range 21-25) in group $2(P=.049)$. RA harvesting time was significantly shorter with the harmonic scalpel technique $(20.3 \pm 3.9$ versus $27.6 \pm 5.4 \mathrm{~min}, P=.022)$. The blood flow of the conduits was similar, with no statistical difference for the 2 arterial conduits (LITA, $P=.76$; RA, $P=.55$ ).

Conclusion: In the learning curve period, the use of a harmonic scalpel is time consuming and presents some difficulties during the harvesting of conduits. According to our

Received fuly 5, 2019; accepted August 2, 2019.

Correspondence: Omer Faruk Dogan, MD, Kafkas University School of Medicine, Department of Cardiovascular Surgery, 36001 Kars, Turkey; $+90-$ 533-4813056; fax: +90-474-2250101 (e-mail: ofdogan@bacettepe.edu.tr). study results, however, the harmonic scalpel technique may be useful because of decreased pathology, including spasm. In our opinion, graft occlusion or thrombus as a life-threatening condition and endothelial dysfunction may decrease with the use of this alternative harvesting technique.

\section{INTRODUCTION}

Arterial conduits in coronary artery bypass grafting (CABG) surgery have a long-lasting impact on survival. Previous publications showed that graft failure due to conduit pathology is related to factors such as harvesting methods in CABG patients [Lehtola 1989; Campeau 1983; Cooper 1996]. During the preparation of arterial conduits by the use of traditional methods, spasm, conduit dissection, or subadventitial hematoma related to mechanical or thermal effects play important roles in bypass graft occlusion [Ronan 2000; Lima Cañadas 2005]. We know that endothelial or subendothelial injury during conduit harvesting is the main reason for histopathologic development and complications in CABG. We showed in our previous study [Dogan 2011] that disruption of intercellular layers and subendothelial and endothelial vacuolization might be the cause of intraluminal coagulation in the early period after CABG. Dysfunction of endothelial integrity may induce atherosclerosis, thrombotic occlusion, and disruption resulting from inhibition of the release of endothelial nitric oxide synthase [Kinoshita 2017; Petry 2012]. Thus, lifethreatening complications may be seen after CABG surgery.

To provide the negative side effects of low-voltage electrocautery such as dissection, endothelial dysfunction, or spasm due to thermal interaction during conduit preparation, the harmonic scalpel technique has been described [Lamm 2000; Orejola 2000; Amaral 1993] and has been suggested as an alternative approach to low-voltage electrocautery use in the preparation of arterial conduits [Lamm 2000; Orejola 2000]. According to the technologic properties of the harmonic scalpel, the mechanical destruction performs the coagulation and cutting process.

To compare the histopathologic changes of endothelial integrity and conduit layers between the 2 harvesting techniques, we examined 2 arterial conduits, the left internal thoracic artery (LITA) and the radial artery (RA), in CABG patients using scanning electron microscopy combined with a 3-dimensional anaglyph method. 
METHODS

After hospital ethics committee approval, informed consent was provided from the patients. A total of 100 patients (59 male) who underwent CABG surgery were included in the study. The patients were divided into groups 1 and 2 with similar preoperative characteristics including mean age, preoperative medications, sex, comorbidities, etc. Patients with chronic liver or kidney disease and hemostatic disorders were excluded. In addition, patients who needed emergent surgery and those with preoperative low cardiac output syndrome, immunologic disorders, and chronic disease were excluded.

All arterial grafts were prepared by experienced surgeons. The preparation of 100 arterial conduits (50 LIMA and $50 \mathrm{RA}$ ) in group 1 was performed with an ultrasonic scalpel (Harmonic Scalpel, Ethicon, Endo-Surgery, Somerville, NJ), which consisted of a generator, cable system, cautery tip, and foot pedal. For coagulation of the side branches, we did not use clips if the diameter of the side branch was $<1.5 \mathrm{~mm}$. In the remaining 50 patients, 50 LIMAs and 50 RAs (100 arterial conduits; group 2) were prepared using low-voltage electrocautery (Valleylab monopolar $300 \mathrm{~W}-300 \mathrm{Ohm}$, Bipolar 70 W-100; Tyco) combined with clips and scissors.

The harmonic scalpel mechanism has been described in previous publications. Briefly, when the foot pedal is pressed and held for 3-4 seconds, the mechanical flow generated by the foot pedal and the longitudinal vibration at the tip of the cautery ensure protein degradation and coagulation. The cautery tip is then turned to cut the coagulated vein with the cutter of the cautery tip.

\section{Techniques of Harvesting and Operations}

In all patients, a complete dose of heparin was given after sternotomy incision. For LITA harvesting in group 1, the endothoracic fascia was cut and dissected from $1 \mathrm{~cm}$ medial using the curved end of the harmonic scalpel. Side branches of LITA with a thickness of $<1.5 \mathrm{~mm}$ were occluded with the harmonic scalpel. If the branches were larger, we used clips. In the classic method in group 2, after a standard midline sternotomy, heparin was administered via central vein before graft harvesting. The LITA was harvested in a pedicled fashion using electrocautery. Next, the branches of the ITA were clipped. In both groups, the terminal portion of the LITA was not cut until perfused. The ITA pedicle was wrapped in a gauze pad soaked with mixed vasodilator solution [Dogan 2006] while blood flow and anastomosis were calculated.

The RA was harvested from the nondominant arm after Allen test in routine surgical fashion. In each group, a sharp incision was made extending from a point lateral to the biceps tendon down to a point approximately $1-2 \mathrm{~cm}$ above the wrist. Conduit dissection and coagulation were provided with the ultrasonically activated harmonic scalpel in group 1 and low-voltage electrocautery in group 2 . We divided the tissues between the arm muscles with careful attention to the cutaneous nerve. In group 1, we dissected with harmonic scalpel to the RA pedicle with satellite veins. In both groups, the tissue was dissected $\geq 1-2 \mathrm{~cm}$ away from the RA. Only
Table 1. Composition of Group 1, Including General Data, Preoperative Hemodynamics, and Medical Treatment*

\begin{tabular}{|c|c|c|c|}
\hline Characteristic & Group 1 & Group 2 & $P$ \\
\hline Patients (n) & 50 & 50 & 1.0 \\
\hline $\operatorname{Sex}(M / F)$ & $33 / 17$ & $31 / 19$ & .85 \\
\hline Age $(y)$ & $56.3 \pm 7.5$ & $59 \pm 9.3$ & .69 \\
\hline Height $(\mathrm{cm})$ & $168(156-188)$ & $163(152-176)$ & .143 \\
\hline Weight (kg) & $76(58-149)$ & $79(62-122)$ & .980 \\
\hline Body mass index $\left(\mathrm{kg} / \mathrm{m}^{2}\right)$ & $24(20-42)$ & $22(19-31)$ & .850 \\
\hline Mean blood pressure $(\mathrm{mm} / \mathrm{Hg})$ & $64 \pm 7$ & $61 \pm 6$ & .086 \\
\hline CPB time (min) & $108 \pm 29$ & $98 \pm 21$ & .790 \\
\hline AoX time (min) & $46 \pm 9.5$ & $43 \pm 6.9$ & .670 \\
\hline Grafted vessels & $4.1 \pm 0.67$ & $3.9 \pm 0.5$ & .66 \\
\hline Smoker (\%) & 39 & 41 & .965 \\
\hline Hypertension (\%) & 42 & 45 & .540 \\
\hline Diabetes (\%) & 36 & 34 & .171 \\
\hline Hypercholesterolemia (\%) & 43 & 46 & .680 \\
\hline$\beta$-Blocker therapy (\%) & 100 & 100 & 1.0 \\
\hline Calcium antagonists (\%) & 85 & 90 & .86 \\
\hline ACE inhibitors (\%) & 55 & 56 & .89 \\
\hline Aggregation inhibitors (\%) & 100 & 100 & 1.0 \\
\hline Anticoagulants (\%) & 7 & 5 & .76 \\
\hline Coronary vasodilators (\%) & 100 & 100 & 1.0 \\
\hline Diuretics (\%) & 20 & 15 & .91 \\
\hline
\end{tabular}

*Data are $\mathrm{n}$, mean $\pm \mathrm{SD}$, or median (range) unless noted otherwise. ACE, angiotensin-converting enzyme; AoX indicates aortic cross-clamp; CPB, cardiopulmonary bypass.

larger branches were occluded using clips and divided by diathermy. We did not use metal clips for smaller branches; instead, we used diathermy alone. Thanks to this approach, we used diathermy at a minimum. We did not cut the distal end of the RAs. The RA conduit was wrapped in a gauze pad soaked with mixed solution while blood flow and anastomosis were calculated. The blood flow of LITAs and RAs was calculated in each patient in both groups during extracorporeal circulation when mean arterial pressure was $>60 \mathrm{mmHg}$. To evaluate the endothelial and subendothelial integrity of each cylinder, a $10-\mathrm{mm}$ length was cut for electron microscopic study.

All operations were performed under extracorporeal circulation. After aortic cross-clamping, cardiac arrest was achieved using antegrade cold cardioplegic solution. After completion of distal and LITA anastomosis, the cross-clamp was released, and proximal anastomosis was performed using an aortic side clamp. No intraaortic balloon pump was used; 11 patients from group 1 and 9 patients from group 2 needed inotropic agents. 
Table 2. According to the Vessel Score System, Histopathology Changes of the Conduits in Both Groups

\begin{tabular}{|c|c|c|c|c|c|}
\hline \multirow[t]{2}{*}{ Criterion } & \multicolumn{4}{|c|}{ Score } & \multirow[t]{2}{*}{ Tota } \\
\hline & 0 & 1 & 2 & 3 & \\
\hline \multicolumn{6}{|l|}{ Group 1} \\
\hline LITA (n) & 47 & 1 & 2 & 0 & 50 \\
\hline LITA (\%) & 94 & 2 & 2 & 0 & \\
\hline RA (n) & 48 & 1 & 1 & 0 & 50 \\
\hline RA (\%) & 96 & 1 & 1 & 0 & \\
\hline Total & 95 & 4 & 6 & 0 & 100 \\
\hline \multicolumn{6}{|l|}{ Group 2} \\
\hline LITA (n) & 39 & 7 & 5 & 4 & 50 \\
\hline LITA (\%) & 78 & 14 & 10 & 8 & 50 \\
\hline RA (n) & 45 & 2 & 2 & 1 & 100 \\
\hline RA (\%) & 90 & 2 & 2 & 0 & \\
\hline Total & 79 & 9 & 10 & 4 & 50 \\
\hline \multicolumn{6}{|l|}{ Total } \\
\hline $\mathrm{n}$ & 79 & 9 & 7 & 5 & 100 \\
\hline$\%$ & 79 & 18 & 14 & 10 & 100 \\
\hline
\end{tabular}

\section{Preparation for Electron Microscopy}

Preparation of the conduit samples has been described [Dogan 2006]. Briefly, the cylinders of both conduits were cut just before anastomosis, immediately washed with a physiologic solution, and immersed in $2.5 \%$ glutaraldehyde for 24 hours. Conduit samples were fixed in $2.5 \%$ glutaraldehyde for 24 hours, washed in phosphate buffer, and postfixed in phosphate buffer $\left(1 \%\right.$ osmium tetroxide $\left.\left[\mathrm{OsO}_{4}\right]\right)$. Samples were dehydrated in concentrated ethyl alcohol, washed with propylene oxide, and placed in epoxy-resin embedding medium. Ultrathin and 2- $\mu \mathrm{m}$ sections were cut with a glass knife ultramicrotome. Semithin sections were stained with methylene blue and examined under a light microscope. Ultrathin sections were collected on copper grids, stained with uranyl acetate and lead citrate, and examined with a JEM 1200EX (Jeol, Tokyo, Japan) electron microscope. A blinded pathologist examined all specimens and described endothelial integrity using the following criteria: (a) completely confluent endothelium; (b) partially confluent endothelium; (c) loosely netted endothelium; (d) islands of endothelium; and (e) no endothelium.

\section{Statistical Analysis}

Statistical analysis was carried out with SPSS 17 for Windows (SPSS, Chicago, IL). Variables are presented as mean \pm standard deviation. To examine the differences in the histopathology and the morphometric parameters for specimens of arterial conduits, we used the paired $t$ test. Comparison of the severity of intimal hyperplasia and atherosclerosis in the RAs was evaluated with the two-sample t test. A $P$ value $<.05$ was considered significant. The normal distribution of variances among groups for continuous variables was evaluated by Levene's test. The

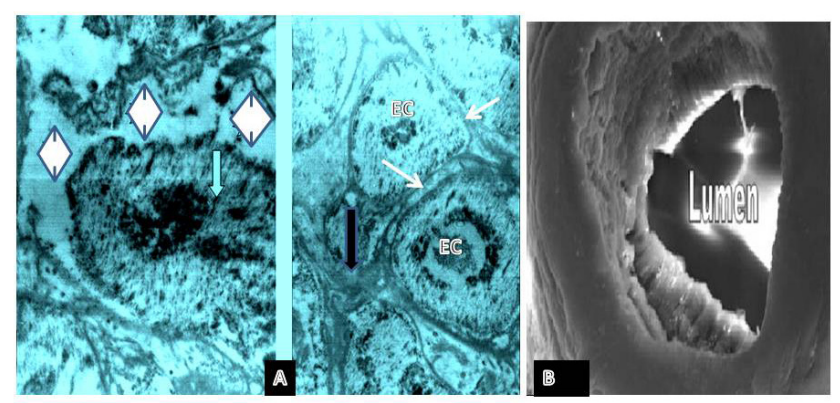

\begin{abstract}
Figure 1. A, Endothelial cell with a heterochromatin-rich nucleus (blue arrow), desquamation, and increasing intercellular gap (white diamonds). Intracellular organelles and cellular interactions are regular. No significant change of intercellular gaps (white arrows) (EC, endothelial cell). B, 3D electron microscopic photograph of the RA demonstrates regular, aligned endothelial layers.
\end{abstract}

chi-square test was used to compare dichotomous variables, and Student's $t$ test was used for continuous variables.

\section{RESULTS}

All the grafts were used in the operations. Pre- and postoperative characteristics of the patients, including aortic cross-clamp time, cardiopulmonary bypass time, number of grafted vessels, the use of inotropic agents, etc., were similar in both groups (Table 1). The mean age of the patients was $59.7 \pm 11.8$ years (range 27-74). Three patients died after surgery (2 from group 1) because of low cardiac output in 2 patients and mediastinitis in 1 patient. The mean number of bypassed coronary arteries was $4.1 \pm 0.67$ and $3.9 \pm 0.50$ in groups 1 and 2, respectively. The harvesting time of LITA was nearly similar in both groups: $26.9 \pm 11.1 \mathrm{~min}$ (range 25-38) in group 1 and 21.3 $\pm 8.6 \mathrm{~min}$ (range 21-25) in group $2(P=.49)$. The duration of RA harvesting time was statistically significantly shorter with the harmonic scalpel technique: $20.3 \pm 3.9 \mathrm{~min}$ in group 1 and $27.6 \pm 5.4 \mathrm{~min}$ in group $2(P=.022)$. In group 1 , we used 8 hemoclips for occluding the conduit branches in only 3 patients ( 3 clips for LITA and 5 clips for RA).

Our study results showed that blood flow did not change significantly regardless of harvesting technique. The mean blood flow of LITA and RA was $70.9 \pm 22.4$ and $92.9 \pm 35.8$ $\mathrm{mL} / \mathrm{min}$ in group 1 , respectively, and $66.3 \pm 12.4$ and $88.4 \pm$ $19.8 \mathrm{~mL} / \mathrm{min}$ in group 2 . There were no significant differences in either arterial conduit (LITA, $P=.76$; RA, $P=.55$ ).

The first 2 days of mediastinal drainage amounted to 826 $\pm 290 \mathrm{~mL}$ (range 485-1100) in group 1. The drainage from the forearm was $16.5 \pm 7.4 \mathrm{~mL}$ (range 14-26). In group 2, the amounts of mediastinal and forearm drainage were 1290 $\pm 520 \mathrm{~mL}$ (range 670-1620) and $30.2 \pm 9.8 \mathrm{~mL}$ (range 25-64) The differences were statistically significant (mediastinum, $P=.034$; forearm, $P=.026$ ).

We used the scoring system described by Yoshikai et al [2004] for histopathologic results: $0=$ no injury; $1=$ mild injury consisting of a slight desquamation in the endothelium 


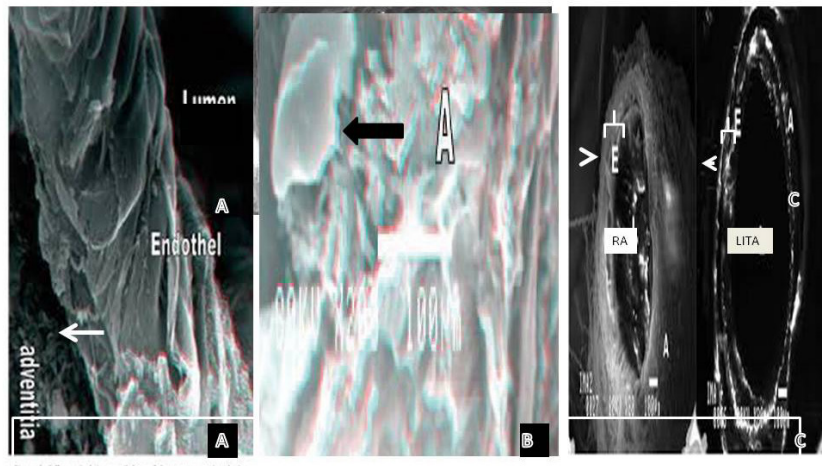

Figure 2. A and B, 3D images of the LITA and the RA, respectively. The white arrow shows separated adventitia from the endothelial layer. This figure also shows endothelial disintegration and distortion, as well as calcification of the medial layer of the RA (black arrow) in combination with a disoriented endothelial layer (B). C, Electron microscopy images of RA and LITA in the harmonic scalpel group.

and minimal exposure in the basal lamina; $2=$ moderate injury, intimal or endothelial discontinuity; and 3 = severe injury, including endothelial separation with endothelial cell loss, basal lamina disruption, and medial edema. According to the vessel scoring system, we summarize the types of histopathologic changes and vessel scores of LITA and RA in Table 2.

We found no histopathologic change in 45 specimens in group 1 (90\% score 0) and 34 specimens in group 2 (68\% score 0$)$. The endothelial surfaces were regular and lined with a normal endothelial layer, and there was no evidence of desquamation of endothelial cells attached to the basal lamina (Figure 1). Intracellular organelles were patent, and intercellular gaps were intact. Pathologic findings of the vascular layers (score 1-3) were detected in 16 conduits in group $2(32 \%)$; disruption of endothelial integrity, and distortion with island-cell like appearance were detected (Figure 2). Vacuoles due to severe edema, especially in the mitochondria, were detected in 4 specimens from group 2. Disintegration of basal lamina of mild to moderate pathology was present in 7 LITA specimens (Figure 3). We detected a different degree of pathology (score 2) in 3 LITA specimens. Endothelial dissection, subendothelial disarrangement, and cellular separation due to intercellular edema were detected as a score 3 pathology in group 2. In 2 RA samples (1 from group 1), medial calcinosis and endothelial hyperplasia were present. No evidence of medial calcinosis or calcification in LITA was reported. In group 1, we detected mild to moderate endothelial or subendothelial pathology in 5 specimens (10\%) (3 LITA, 6\%; 2 RA, 4\%). No severe pathology was detected in group 1 patients. There was a significant difference in pathologic changes between groups $(P=.001)$.

\section{DISCUSSION}

In total arterial revascularization, harvesting of the ITA and the RA are being increasingly used in CABG patients.
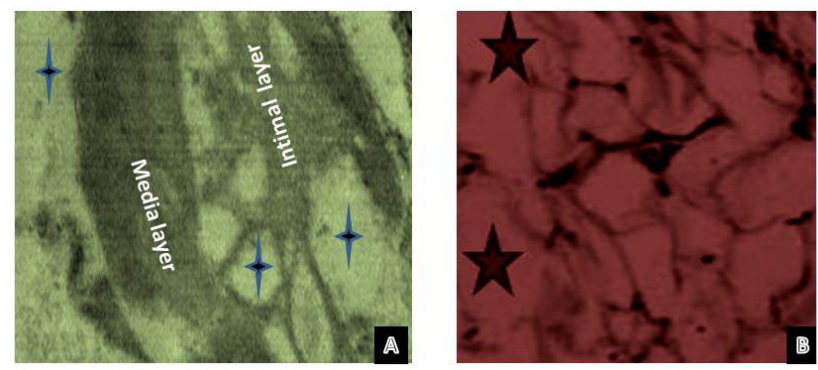

Figure 3. Score 3 pathology in a LITA specimen. A, Endothelial dissection, subendothelial disarrangment, and cellular separation are detected (blue stars). Also shown are disintegration of the intimal layer, endothelial cell disarrangment, and severe separation of the left internal thoracic artery because of edematous changes. B, Pathology of tunica media in the RA, with vacuoles.

Recent trials have shown that despite careful attention during harvesting of conduits using either method, spasm and vessel pathology of the RA and LITA have been detected in CABG surgery [Dogan 2006; Higami 2000a; Fukata 2002]. The true prevalence of pathologic changes and spasm of the arterial conduits is unknown. Because of endothelial or subendothelial damage and disruption of conduits, such as intraluminal thrombus formation, dissection, disarrangement of conduits' endothelial layers, and atherosclerosis, which lead to lifethreatening conditions during harvesting, some alternative devices and techniques have been described [Higami 2000b; Posacıoglu 1998]. One of these is the ultrasonically activated harmonic scalpel [Posacıoğlu 1998; Takao 2000]. Frequently, the harmonic scalpel as a new medical device is used for cutting and coagulating with high-frequency ultrasonic vibrations in laparoscopic surgery. Unlike electrocautery, the harmonic scalpel can be used closer than $1 \mathrm{~mm}$ from vascular tissue without any hemorrhagic complication during tissue dissections in a number of surgical procedures [Takao 2000; Ohtsuka 1998; Onan 2014].

Pathologic changes of the arterial conduits, including endothelial disintegration, corruption, or dissection and spasm related to thermal injury, have been reported in a number of investigations [Ohtsuka 1998; Onan 2014]. Endothelial complications as a result of the use of electrocautery have been reported by Onan et al [2014]. They suggested a sharp dissection method during ITA mobilization to ensure endothelial integrity of the ITA conduit rather than electrocautery [Onan 2014]. Therefore, different techniques and devices have been proposed to decrease endothelial injury, harvesting time, and frequency of spasm. To provide low flow from the conduits and suitable endothelial integrity, the harmonic scalpel instead of electrocautery with hemoclips is one of the instrumental improvements in CABG surgery [Tanemoto 1998]. Although it has been suggested that this technique improves endothelial integrity, other studies have failed to confirm this finding [Higami 2000a, b].

To explain this important problem, we investigated the differences of side effects of the harmonic scalpel and electrocautery on histopathologic changes, and we compared pathologic complications of the 2 techniques. Our investigation 
demonstrates for the first time that harvesting of LITA and the RA using the harmonic scalpel is associated with better preservation of endothelial and subendothelial integrity compared with the standard harvesting method. In the harmonic scalpel group, we detected endothelial or subendothelial pathology in only 5 specimens. According to our investigation, this technique decreased the histopathologic changes of conduits significantly compared with low-voltage electrocautery. We clearly confirmed the pathologic changes using electron microscopic examination, which showed disarrangement and severe edema of endothelial or subendothelial layers in group 1 patients. In the classic harvesting method, pathologic findings of the vascular layers were higher in LITA than RA. Endothelial dissection, subadventitial hematoma, subendothelial disarrangement, and cellular separation due to intercellular edema were the main pathologic changes in group 2. Additionally, edematous intracellular organelles in the samples with severe tunica media pathology were detected. Our study results show that low-voltage electrocautery increased the intercellular gap in the LITA samples. Loss of endothelial cells from the external elastic membrane and tunica adventitia was present in 3 RA and 7 LITA specimens.

In contrast to our investigation, no endothelial damage due to the harmonic scalpel was reported by Yoshikai et al [2004]; that study, however, included only 9 patients. In our opinion, because our study included more samples, we detected pathologic changes of the LITA and the RA in 5 patients in the harmonic scalpel group. Discontinuity of the vascular wall structures and tissue denaturation on the ITA was reported by Higami et al [2000a]. They suggested that branches should be sectioned $\geq 1 \mathrm{~mm}$ distal to their origin for secure skeletonization of ITA using an ultrasonic scalpel [Higami 2000a]. Because it creates coagulation and protein denaturation directly to the tissue through tissue destruction, the harmonic scalpel causes fewer changes in the surrounding tissue and conduits. Thanks to these advantages, there was less damage to endothelial integrity in the prepared arterial grafts in group 1 patients.

The electrocautery technique is frequently used by surgeons for harvesting both conduits. Unfortunately, it has been demonstrated that when the electrocautery contacts the hemostatic clips, mural thrombus formation, dissection, and damage of endothelial integrity could be present [Thatte 2001; Verrier 1996; Lehtola 1989]. Because the harmonic scalpel device is used in a limited number of CABG patients, very little is known about its effects on endothelial and subendothelial integrity and histologic changes the arterial bypass conduits. Tanemoto et al [1998] showed that there was less tissue damage during harmonic scalpel use in animal models. They suggested that the lower thermal effect was the important factor during harvesting. Indeed, in contrast to our investigation, histological examination of the arterial graft preparation using the ultrasonic scalpel did not show any damage to the arterial wall when it accidentally contacted the arterial conduit [Tanemoto 1998; Thatte 2001; Lehtola 1989; Lamm 2000]. We believe that these differences may be related to differences in the number of conduits been investigated using an electron microscope for ultrastructural analyses.
We did detect low cardiac output syndrome related to graft spasm postoperatively with electrocardiographic changes in 5 patients; the standard harvesting method was used in 4 of the 5. We replaced the LITA using a saphenous vein in these patients.

The tip handle of the harmonic scalpel presents some difficulties for delicate maneuvers during LITA harvesting because it is much longer than the classic electrocautery tool. Therefore, this drawback of the harmonic scalpel may increase the time needed to prepare LITA in the beginning of the learning curve. According to our study, the tip of the harmonic scalpel should be used $\geq 1-2 \mathrm{~mm}$ away from the arterial grafts.

The main disadvantage of the harmonic scalpel is its price, which is higher than standard electrocautery. However, we used resterilized harmonic scalpel tips to provide significant economic savings.

In conclusion, use of the harmonic scalpel seems to ensure endothelial integrity of arterial conduits, less severe spasm, and less damage of conduit layers during harvesting compared with standard low-voltage electrocautery. Because this method does not cause thermal endothelial injury, it may prevent unexpected life-threatening complications and increase the long-term graft patency rate.

\section{ACKNOWLEDGEMENT}

The authors would like to thank Prof Dr. Omer Faruk Dogan, who supported the planning and preparation of research and rewriting the manuscript in the revision process.

\section{REFERENCES}

Amaral JF. Comparison of the ultrasonically activated scalpel to electrosurgery and laser for laparoscopic surgery. Surg Endosc 1993;7:141-142.

Campeau L, Enjalbert M, Lesperance J, et al. Atherosclerosis and late closure of aortocoronary saphenous vein grafts: Sequential angiographic studies at 2 weeks, 1 year, 5 to 7 years, and 10 to 12 years after surgery. Circulation 1983;68:1-7.

Cooper G, Underwood M, Deverall P. Arterial and venous conduits for coronary artery bypass. A current review. Eur J Cardiothorac Surg 1996;10:129-140.

Dogan OF, Tatar I, Duman U, Yorgancioglu C, Demircin M, Aldur M, Celik HH, Boke E. Comparison of the pretreatment effects of mixed vasodilators (3-D solution) on radial and internal thoracic arteries by using a 3-dimensional (3D) anaglyph electron microscope technique. Heart Surg Forum 2006;9:643-649.

Dogan OF. Examination of the internal thoracic artery by transmission electron microscope in coronary artery bypass surgery patients. Turk J Thorac Cardiovasc Surg 2011;19:329-336.

Fukata Y, Horike K, Kano M. Histological study on the influences of an ultrasonic scalpel on skeletonized vessel wall. Ann Thorac Cardiovasc Surg 2002;8:291-297.

Higami T, Kozawa S, Asada T, et al. Skeletonisation and harvest of the internal thoracic artery with an ultrasonic scalpel. Ann Thorac Surg 2000;70:307-308. 
Higami T, Maruo A, Yamashita T, Shida T, Ogawa K. Histologic and physiologic evaluation of skeletonized internal thoracic artery harvesting with an ultrasonic scalpel.J Thorac Cardiovasc Surg 2000;120:1142-1147.

Kinoshita T, Tawa M, Suzuki T, Aimi Y, Asai T, Okamura T. Endothelial dysfunction of internal thoracic artery graft in patients with chronic kidney disease. J Thorac Cardiovasc Surg 2017;153:317-324.

Lamm P, Juchem G, Weyrich P, Schütz A, Reichart B. The harmonic scalpel: optimizing the quality of mammary artery bypass grafts. Ann Thorac Surg 2000; 69:1833-1835.

Lehtola A, Verkkala K, Jarvinen A. Is electrocautery safe for internal mammary artery mobilisation? A study using scanning electron microscopy. Thorac Cardiovasc Surgeon 1989;37:55-57.

Lima Cañadas PP, Cañas AC, Orradre Romeo JL, Rubio Martínez CI, López Almodóvar LF, Calleja Hernández M. Endothelium histological integrity after skeletonized dissection of the left internal mammary artery with ultrasonic scalpel. Interact Cardiovasc Thorac Surg 2005;4:160-162.

Ohtsuka T, Wolf KR, Warnig P, Park SE. Thoracoscopic limited pericardial resection with an ultrasonic scalpel. Ann Thorac Surg 1998;65:855-856.

Onan B, Yeniterzi M, Onan IS, Ersoy B, Gonca S, Gelenli E, Solakoglu S, Bakir I. Effect of electrocautery on endothelial integrity of the internal thoracic artery: ultrastructural analysis with transmission electron microscopy. Tex Heart Inst J 2014;1;41:484-490.

Orejola WC, Villacin AB, Defilippi VJ, Mekhjian HA. Internal mammary artery harvesting using the harmonic scalpel. ASAIO J 2000;46:99-102.
Petry A, BelAiba RS, Weitnauer M, Görlach A. Inhibition of endothelial nitric oxide synthase increases capillary formation via Rac1-dependent induction of hypoxia-inducible factor- $1 \alpha$ and plasminogen activator inhibitor-1. Thromb Haemost 2012;108:849-862.

Posacıŏglu H, Atay Y, Çetindağ B, et al. Easy harvesting of radial artery with ultrasonically activated scalpel. Ann Thorac Surg 1998;65:984-985.

Ronan WJ, Perry AL, Barner BH, et al. Radial artery harvest: Comparison of ultrasonic dissection with standard technique. Ann Thorac Surg 2000;69:113-114.

Takao S, Shinchi H, Maemura K, Aikou T. Ultrasonically activated scalpel is an effective tool for cutting the pancreas in biliary-pancreatic surgery: experimental and clinical studies. J Hepatobiliary Pancreat Surg 2000;7:58-62.

Tanemoto K, Kanaoka Y, Murakami T, et al. Harmonic scalpel in coronary artery bypass surgery. J Cardiovasc Surg 1998;39:493-495.

Thatte HS, Khuri SF. The coronary artery bypass conduit: I. Intraoperative endothelial injury and its implication on graft patency. Ann Thorac Surg 2001;72:2245-2252

Verrier ED, Boyle EM Jr. Endothelial cell injury in cardiovascular surgery. Ann Thorac Surg 1996;62:915-922.

Yoshikai M, Ito T, Kamohara K, Yunoki J. Endothelial integrity of ultrasonically skeletonized internal thoracic artery: Morphological analysis with scanning electron microscopy. Eur J Cardiothorac Surg 2004;25:208-211. 